\title{
Synthesis and Antibacterial Activity of Urea and Thiourea Derivatives of Anacardic Acid Mixture Isolated from A Natural Product Cashew Nut Shell Liquid (CNSL)
}

\author{
N. Subhakara Reddy ${ }^{1,2}$, A. Srinivas Rao ${ }^{1 *}$, M. Adharvana Chari ${ }^{3}$, V. Ravi kumar ${ }^{1}$, \\ V. Jyothy ${ }^{3}$, V. Himabindu ${ }^{2}$ \\ ${ }^{1}$ Medicinal Chemistry Laboratory, GVK Biosciences Pvt. Ltd., Hyderabad, India \\ ${ }^{2}$ Centre for Environment, Institute of Science and Technology, JNT University, Hyderabad, India \\ ${ }^{3}$ Dr. MACS Bio-Pharma Pvt. Ltd., Westren Hills, Hyderabad, India \\ Email: ${ }^{*}$ machari100@yahoo.com
}

Received May 10, 2012; revised June 25, 2012; accepted July 4, 2012

\begin{abstract}
Synthesis and antibacterial activity of some novel urea and thiourea derivatives (8a - 8k, 9a - 9f) of anacardic acid prepared from commercially available anacardic acid which is obtained from natural product Cashew Nut Shell Liquid (CNSL). Compounds ( $8 \mathrm{a}-8 \mathrm{k}, 9 \mathrm{a}-9 \mathrm{f})$ were tested for Gram positive and Gram negative bacterial cultures. Most of the compounds were showed active compared with standard drug ampicilline.
\end{abstract}

Keywords: Synthesis; Urea and Thiourea Derivatives; Anacardic Acid; Anti-Bacterial Activity

\section{Introduction}

Anacardic acid mixture (1a-d) isolated from a natural product Cashew Nut Shell Liquid (CNSL) which is a by-product of cashew nut industry and these are salicylic acid derivatives with a nonisoprenoid alk(en)yl side chain [1]. Kubo et al. reported the separation of anacardic acid into monoene (15:1), diene (15:2) and triene $(15: 3)$ by preparative HPLC and tested against cancer cells, and found to show moderate cytotoxic activity on BT-20 breast and HeLa epithelioid cervix carcinoma cells [2]. The emergence of drug resistant strains in clinical applications [3-5] especially to Gram positive bacteria $[6,7]$ has created a problem of global proportions $[8,9]$. This phenomenon has led in creating novel antibacterial agents distinct from existing classes of compounds. Anacardic acid (pentadecyl salicylic acid) is a phenolic constituent present in Cashew Nut Shell Liquid (CNSL); (Anacardium occidentale L.) and exhibits antimicrobial properties $[10,11]$ which have led to the preparation of various analogues $[12,13]$. Anacardic acid and its derivatives exhibits biological activities like antimicrobial activity $[10,11]$ and soybean lipoxygenase-1 inhibitory activity[14,15]. G. C. Reddy et al. reported the synthesis of benzamide derivatives of anacardic acid [16], sildenafil analogues [17], dihydropyridine analogues [18] as calcium channel blockers, isonicotinoylhydrazones for

${ }^{*}$ Corresponding author. antimycobacterial activity [19] starting from anacardic acid. Recently, a few anacardic acid derivatives exhibited various activities like affect the structure of the enzyme [20], anacardic acid is a specific activator of kinase activity of Aurora Kinase A [21], suppresses expression of nuclear factor-kB regulated gene products leading to potentiation of apoptosis [22] inhibitor of the HAT activity of recombinant Plasmodium falciparum GCN5 [23] and as modulators of histone acetyltransferases [24].

In the present work we wish to report to synthesize novel cell permeable urea and thiourea compounds from cheaply available anacardic acid which was a major constituent of Cashew Nut Shell Liquid (CNSL) natural source to evaluate their biological activity by various antibacterial strains. This report describes the synthesis, spectroscopic identification and antibacterial activity of some novel urea and thiourea anacardic acid derivatives against Escherichia coli, Pseudomonas aeruginosa, Staphylococcus aureus and Streptococcus pyogenes bacterial strains.

\section{Results and Discussion}

Here we described the synthesis of various biologically active novel urea and thiourea derivatives using anacardic acid mixture as starting material and various reagents in the given below conditions (Scheme 1).

The anacardic acid mixture (1a-d) was isolated from commercially available CNSL by a reported method [25]. 


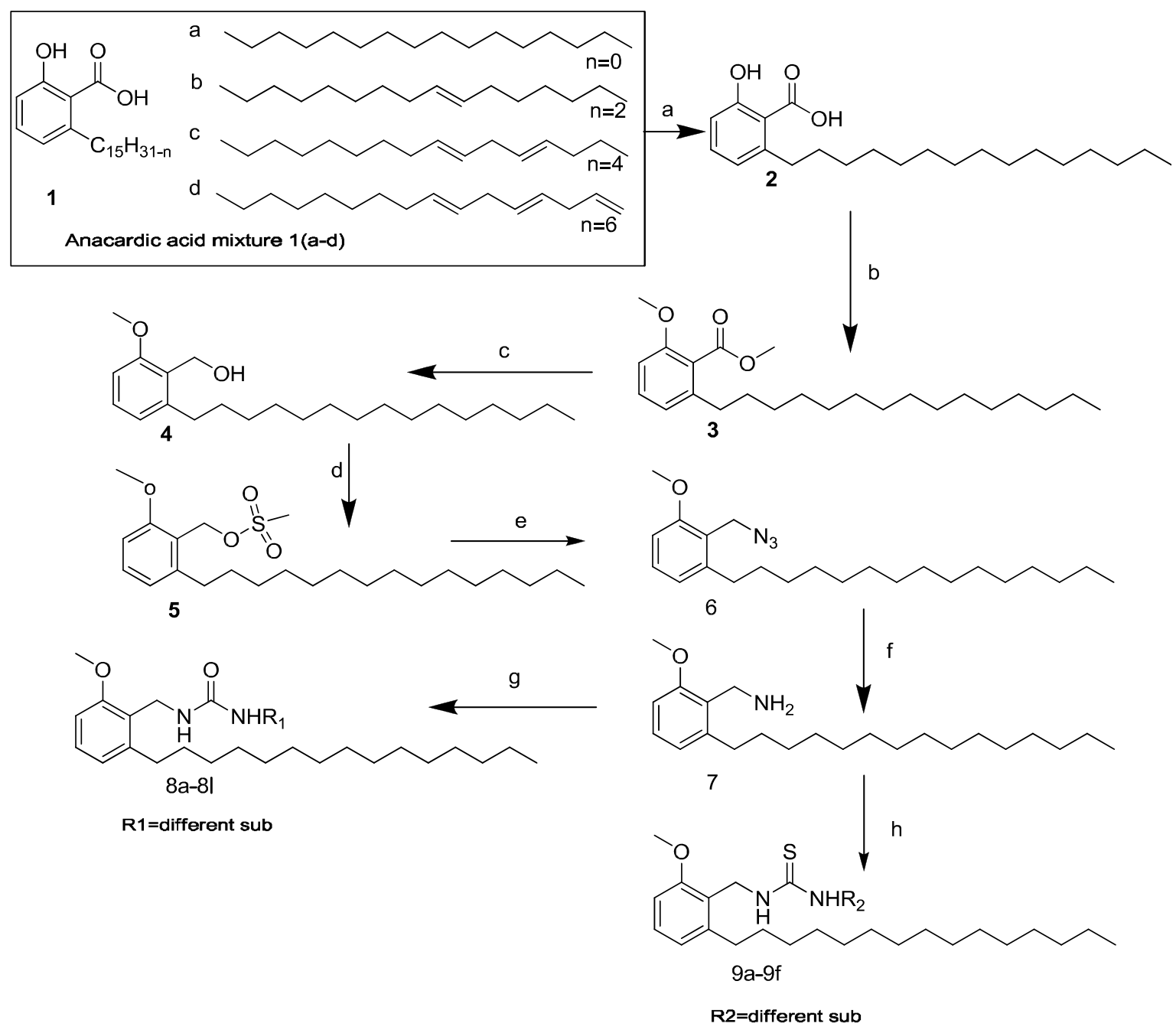

Reagents: a) $10 \% \mathrm{Pd} / \mathrm{C}$, EtOH, 50psi, RT, 2 hrs; b) Dimethyl sulfate, $\mathrm{K}_{2} \mathrm{CO}_{3}$, Acetonitrile, $90^{\circ} \mathrm{C}, 24$ hrs; c) LAH, THF, $0^{\circ} \mathrm{C}$-rt, 18 hrs; d) Methane sulphonyl chloride, $\mathrm{Et}_{3} \mathrm{~N}$, DCM, $0^{\circ} \mathrm{C}$-rt, $3 \mathrm{hrs}$; e) $\mathrm{NaN}_{3}$, DMF; f) $10 \% \mathrm{Pd} / \mathrm{C}, 50 \mathrm{psi}, 2 \mathrm{hrs}$; g) different isocynate, $\mathrm{CHCl}_{3}$, h) different isothiocynate, $\mathrm{CHCl}_{3}$.

Scheme 1: Synthesis of various biologically active urea and thiourea derivatives from anacardic acid mixture.

Accordingly CNSL was treated with calcium hydroxide, during which anacardic acid present in CNSL becomes calcium anacardate, which was isolated and hydrolyzed with dil. hydrochloric acid to generate anacardic acid ene mixture, which was a mixture of monoene, diene and triene located at $\left(8^{\prime}\right),\left(8^{\prime}, 11^{\prime}\right)$ and $\left(8^{\prime}, 11^{\prime}, 14^{\prime}\right)$ of the C15 alkyl chain respectively. Saturated anacardic acid was obtained by hydrogenation of the ene mixture of anacardic acid [17] and was further converted to dialkylated compound by reacting with dimethylsulfate in acetonitrile. Dialkylated anacardic acid was reduced to alcohol by treatment with lithium aluminium hydride in tetrahydrofuran and then protected with methane sulphonyl chloride in dichloromethane. Resultant mesylated compound was reacted with sodium azide followed by reduction with $\mathrm{Pd} / \mathrm{C}$ under $\mathrm{H}_{2}$ pressure to obtained amine coupled with various isocynate or iso thiocynate in chloroform to obtain compounds ( $8 \mathrm{a}-8 \mathrm{k}, 9 \mathrm{a}-9 \mathrm{f}$, Scheme 1) of urea and thiourea derivatives were purified by column chromatography to yield title compounds. The structure of urea and thiourea derivatives $(8 \mathrm{a}-8 \mathrm{k}, 9 \mathrm{a}-9 \mathrm{f})$ was determined by using different spectroscopic techniques ${ }^{1} \mathrm{H}$ NMR, IR, Mass. The resulting compounds are screened for their antibacterial activity.

Biological Activity: The urea and thiourea derivatives (8a - 8k,9a - 9f) were screened for their antibacterial activity[26] against some of the pathogenic bacteria viz. E.coli (MTCC443), P. aeruginosa (MTCC424), S. aureus, (MTCC96) and S. pyogenes (MTCC443) using agar well diffusion method according to the literature protocol [26] The anti-bacterial activity of the analogues was compared with standard drug ampicilline and the results of investigation have been presented in Table 1 and observed that some of the compounds are showed high biological activity. Based on the test results it is evident that several of synthesized anacardic acid analogues 
Table 1. Antibacterial activity of Urea and Thiourea derivatives of anacardic acid.

\begin{tabular}{|c|c|c|c|c|c|}
\hline \multirow[b]{2}{*}{ Compound No. } & \multirow[b]{2}{*}{$\mathbf{R}$} & \multicolumn{4}{|c|}{ Name of the Bacteria (Conc. $250 \mu \mathrm{g} / \mathrm{ml}$ ) \& Inhibition Zone in $\mathrm{mm}$} \\
\hline & & $\begin{array}{c}\text { E. coli } \\
\text { MTCC443 }\end{array}$ & $\begin{array}{c}\text { P. aeruginosa } \\
\text { MTCC424 }\end{array}$ & $\begin{array}{l}\text { S. aureus } \\
\text { MTCC96 }\end{array}$ & $\begin{array}{l}\text { S. pygenes } \\
\text { MTCC442 }\end{array}$ \\
\hline $\mathrm{S}^{*}$ ampicilline & SD* amplicilline & 20 & 20 & 18 & 19 \\
\hline $8 \mathrm{a}$ & & 19 & 18 & 17 & 16 \\
\hline $8 \mathrm{~b}$ & & 22 & 20 & 16 & 17 \\
\hline $8 \mathrm{c}$ & & 20 & 20 & 16 & 16 \\
\hline $8 \mathrm{~d}$ & & 18 & 16 & 16 & 18 \\
\hline $8 \mathrm{e}$ & & 24 & 20 & 17 & 17 \\
\hline $8 \mathrm{f}$ & & 25 & 18 & 17 & 17 \\
\hline $8 \mathrm{~g}$ & & 18 & 17 & 16 & 16 \\
\hline $8 \mathrm{~h}$ & & 23 & 17 & 15 & 19 \\
\hline $8 \mathrm{i}$ & & 24 & 19 & 17 & 18 \\
\hline $8 \mathrm{j}$ & & 23 & 17 & 17 & 17 \\
\hline $8 \mathrm{k}$ & & 15 & 15 & 15 & 16 \\
\hline $9 \mathrm{a}$ & & 15 & 16 & 17 & 18 \\
\hline $9 b$ & & 17 & 19 & 17 & 17 \\
\hline
\end{tabular}


$9 \mathrm{c}$

$9 \mathrm{~d}$

$9 \mathrm{e}$

$9 f$

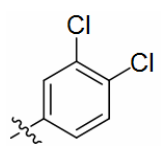<smiles>Fc1ccccc1</smiles><smiles>N#Cc1ccccc1</smiles><smiles>FC(F)(F)c1cccc(C(F)(F)F)c1</smiles>

18 possess moderate to good activity against the Gram + ve and Gram - ve bacteria. Of all the compounds prepared entities $8 \mathrm{~h}, 8 \mathrm{i}, 8 \mathrm{j}, 9 \mathrm{e}$ showed good activity against $E$. coli, $8 \mathrm{~b}, 8 \mathrm{c}, 8 \mathrm{e}$ and $8 \mathrm{f}$ of $E$. coli MTCC443, and of $P$. aeruginosa MTCC424, display good to excellent activity while the remaining compounds showed moderate activity. The most active antibacterial agent against Escherichia coli found to be compound $8 \mathrm{~h}, 8 \mathrm{i}, 8 \mathrm{j}$, and $9 \mathrm{e}$ having $-\mathrm{CN}$, $-\mathrm{CF}_{3}$ and $-\mathrm{OCH}_{3}$ groups and other compounds in the series exhibited moderate to good activity. The compound 9b, 9c, 9d and 9e showed good activity against almost all the strains and 9 f also showed good activity against $S$. aureus MTCC96 and $S$. pyogenes MTCC442. This indicates chloro, dichloro, cyano and methoxy substituted compounds showed better activity when compared to other substituted groups. Here seems to be, thiourea substituted novel compounds are exhibiting better activity than urea substituted compounds. The activity depends to some extent on the $\mathrm{R}$ substituent, however all the compounds showed antibacterial activity. So other functionalities in the molecule will contribute to the activity as well. It may be suggested that the anacardic acid derivative with a suitable R may lead to a good antibacterial agent against all the Escherichia coli, Pseudomonas aeruginosa, Staphylococcus aureus and Streptococcus pyogenes bacterial strains.

\section{Conclusion}

In summary, the present study describes a convenient and efficient protocol for the synthesis of sulfonamide derivatives by using anacardic acid mixture using various reagents and different conditions. We believe that this procedure is convenient, economic and a user- friendly process for the synthesis of these various novel urea and thiourea compounds from anacardic acid mixture. All compounds structures are supported by physico chemical and IR, NMR, Mass spectral data. Urea and thiourea derivatives were screened for their antibacterial activity against few bacterial strains and observed that some of the compounds are showed more biological activity than standards used.

\section{Experimental Section}

\subsection{General Reagents and Equipment}

All chemicals and solvents were obtained from Aldrich and Spectrochem., India and used without further purification. Column chromatographic separations were carried out on silica gel 60 - 120 mesh size. Melting points were determined in open glass capillaries on a Mel-temp apparatus and are uncorrected. The IR spectra were recorded on a Thermo Nicolet IR 200 FT-IR spectrometer as $\mathrm{KBr}$ pellets and the wave numbers were given in $\mathrm{cm}^{-1}$. The ${ }^{1} \mathrm{H}$ and ${ }^{13} \mathrm{C}$ NMR spectra of samples were recorded on a Varian EM-360, NMR spectrometer using TMS as an internal standard in $\mathrm{CDCl}_{3}$. The mass spectra were recorded on Jeol JMS-D 300 and Finnigan Mat b at 70 $\mathrm{eV}$ with an emission current of $100 \mu \mathrm{A}$.

Synthesis of 2-hydroxy-6-pentadecyl-benzoic acid (2): A solution of compound 1 (100 g, $287.35 \mathrm{mmol})$ in ethanol $(500 \mathrm{ml})$ was taken into a $1 \mathrm{~L}$ Parr-hydrogenation vessel and added a suspension of $10 \% \mathrm{Pd} / \mathrm{C}(10 \mathrm{~g}, 10 \%)$ in $70 \mathrm{ml}$ of ethanol under argon atmosphere and applied $\mathrm{H}_{2}$-pressure (50 psi) for $2 \mathrm{~h}$. Reaction mixture was filtered through celite bed and concentrated the filtrate under reduced pressure to obtained compound recrystallized in pet ether to get 2-hydroxy-6-pentadecyl-benzoic acid (2) as white color solid (70 g); (Yield: $70 \mathrm{~g}, 68.8 \%$; white solid); M.p: $85^{\circ} \mathrm{C}-86^{\circ} \mathrm{C}$; IR (KBr): $v_{\max } 3010,3002$, 
2918, 2851, 1655, 1450, 1246, $1214 \mathrm{~cm}^{-1} ; \mathrm{H}^{1}$-NMR (400 $\mathrm{MHz}, \mathrm{CDCl}_{3}$ ): $\delta 0.89(\mathrm{t}, 3 \mathrm{H}, J=6.8 \mathrm{~Hz}), 1.27$ (brs, 24H), $1.57-1.63(\mathrm{~m}, 2 \mathrm{H}), 2.98(\mathrm{t}, 2 \mathrm{H}, J=8 \mathrm{~Hz}), 6.78(\mathrm{~d}, 1 \mathrm{H}, J$ $=7.6 \mathrm{~Hz}), 6.88(\mathrm{~d}, 1 \mathrm{H}, J=8.4 \mathrm{~Hz}), 7.37(\mathrm{t}, 1 \mathrm{H}, J=8 \mathrm{~Hz})$, 11.02 (bs, 1H); EI MS: m/z (rel.abund.\%) $349\left(\mathrm{M}^{+}, 100\right)$.

Synthesis of 2-methoxy-6-pentadecyl-benzoic acid methyl ester (3): A solution of compound 2 (20 g, $57.471 \mathrm{mmol})$ in ACN $(200 \mathrm{ml})$ was added $\mathrm{K}_{2} \mathrm{CO}_{3}(40.8$ g, $287.35 \mathrm{mmol})$ and DMS (21.76 ml, $229.88 \mathrm{mmol})$. The content was heated refluxed for $24 \mathrm{hrs}$. Reaction mixture was filtered and distilled off filtrate, obtain residue was redissolved in ethyl acetate and washed with water $(2 \times 200 \mathrm{ml})$, brine solution $(175 \mathrm{ml})$, dried over anhydrous $\mathrm{Na}_{2} \mathrm{SO}_{4}$, filtered and evaporated under vacuum to obtain 2-Methoxy-6-pentadecyl-benzoic acid methyl ester (3) as pale yellow solid $18.5 \mathrm{~g}$; Yield: $85.5 \%$; M.p: $36^{\circ} \mathrm{C}-37^{\circ} \mathrm{C}$; IR (KBr): $v_{\max } 3004,2921$, 2852, 1732, 1589, 1460, 1266, 1105, 1067, 954, 747 $\mathrm{cm}^{-1} ; \mathrm{H}^{1}$-NMR $\left(400 \mathrm{MHz}, \mathrm{CDCl}_{3}\right): \delta 0.88(\mathrm{t}, 3 \mathrm{H}, J=6.8$ $\mathrm{Hz}), 1.25$ (brs, 24H), $1.53-1.60(\mathrm{~m}, 2 \mathrm{H}), 2.53$ (t, 2H, $J=$ $8 \mathrm{~Hz}), 3.81$ (s, 3H), 3.90 (s, 3H), $6.75(\mathrm{~d}, 1 \mathrm{H}, J=8.4 \mathrm{~Hz})$, $6.82(\mathrm{~d}, 1 \mathrm{H}, J=8 \mathrm{~Hz}), 7.25(\mathrm{t}, 1 \mathrm{H}, J=8 \mathrm{~Hz}) ;{ }^{13} \mathrm{C} \mathrm{NMR}$ $\left(100 \mathrm{MHz} \mathrm{CDCl}_{3}\right): \delta$ 14.07, 22.66, 29.32, 29.38, 29.48, $29.51,29.61,29.65,31.12,31.88,33.45,52.04,55.80$, 108.30, 121.45, 123.44, 130.17, 141.35, 156.20, 168.88; EI MS: m/z (rel.abund.\%) $377\left(\mathrm{M}^{+}, 100\right)$.

Synthesis of 2-Methoxy-6-pentadecyl-phenyl)-methanol (4): A suspension of $\mathrm{LiAlH}_{4}$ (3.03 g, 79.787 $\mathrm{mmol})$ in Dry THF $(150 \mathrm{ml})$ was added compound 3 (20 $\mathrm{g}, 53.19 \mathrm{mmol})$ in THF $(100 \mathrm{ml})$ drop wise over a period of $40 \mathrm{~min}$ at $0^{\circ} \mathrm{C}$. The content was slowly bringing it to room temp., stirred at room temp., for $18 \mathrm{~h}$. Reaction mixture was quenched with saturated brine solution (40 $\mathrm{ml})$ at $0^{\circ} \mathrm{C}$ diluted with ethyl acetate filtered $(200 \mathrm{ml})$ and washed with ethyl acetate $(100 \mathrm{ml})$, filtrate was washed with brine solution $(200 \mathrm{ml})$ dried over anhydrous $\mathrm{Na}_{2} \mathrm{SO}_{4}$, filtered and evaporated under vacuum to obtain 2-Methoxy-6-pentadecyl-phenyl)-methanol (4) (15 gm) as off white solid; Yield: $81 \%$; M.p. $60^{\circ} \mathrm{C}-62^{\circ} \mathrm{C}$; IR (KBr): $v_{\max } 3386,3073,2917,2847,1589,1465,1318$, 1263, 1197, 1093, 1001, $739 \mathrm{~cm}^{-1}$; $\mathrm{H}^{1}$-NMR (400 MHz, $\left.\mathrm{CDCl}_{3}\right): \delta 0.89(\mathrm{t}, 3 \mathrm{H}, J=7.2 \mathrm{~Hz}), 1.27(\mathrm{brs}, 24 \mathrm{H}), 1.53-$ $1.58(\mathrm{~m}, 2 \mathrm{H}), 2.37(\mathrm{t}, 1 \mathrm{H}, J=6.4 \mathrm{~Hz}), 2.68(\mathrm{t}, 2 \mathrm{H}, J=$ $6.4 \mathrm{~Hz}), 3.87(\mathrm{~s}, 3 \mathrm{H}), 4.75(\mathrm{~d}, 2 \mathrm{H}, J=6.4 \mathrm{~Hz}) 6.77$ (d, $1 \mathrm{H}, J=8 \mathrm{~Hz}), 6.82(\mathrm{~d}, 1 \mathrm{H}, J=7.6 \mathrm{~Hz}), 7.20(\mathrm{t}, 1 \mathrm{H}, J=8$ $\mathrm{Hz}) ;{ }^{13} \mathrm{C}$ NMR $\left(100 \mathrm{MHz}, \mathrm{CDCl}_{3}\right): \delta 14.07,22.66,29.32$, 29.46, 29.57, 29.61, 29.65, 31.88, 32.10, 33.19, 55.37, 57.30, 108.01, 122.28, 126.83, 128.45, 142.62, 158.22; EI MS: m/z (rel.abund.\%) $349\left(\mathrm{M}^{+}, 100\right)$.

Synthesis of methanesulfonic acid 2-methoxy-6-pentadecyl-benzyl ester (5): To a solution of compound 4 $(15 \mathrm{~g}, 45.181 \mathrm{mmol})$ in DCM was added TEA (13.92 $\mathrm{ml}$, $99.398 \mathrm{mmol}$ ) followed by mesyl chloride $(4.2 \mathrm{ml}, 54.21$ mmol) at $0^{\circ} \mathrm{C}$ over a period of 15 mins. The content was stirred at room temp., for $3 \mathrm{hrs}$. Reaction mixture was washed with water $(150 \mathrm{ml})$, brine solution $(200 \mathrm{ml})$ dried over anhydrous $\mathrm{Na}_{2} \mathrm{SO}_{4}$, filtered and evaporated under vacuum to obtain methanesulfonic acid 2-methoxy -6-pentadecyl-benzyl ester (5) (17 g, 92.59\%) as offwhite color solid.

Synthesis of 2-(azidomethyl)-1-methoxy-3-penta-decyl-benzene (6): To a solution of compound 5 (7.5 g, $17.605 \mathrm{mmol})$ in DMF $(30 \mathrm{ml})$ was added sodium azide (1.49 g, $22.887 \mathrm{mmol})$, the content was heated at $100^{\circ} \mathrm{C}$ for 2 hrs. Reaction mixture was cooled to room temp., and poured into cool water $(150 \mathrm{ml})$ solid compound was precipitated filtered and dried to get Azide (6) (6.2 g, 94.4\%) as white color solid; IR (KBr): $v_{\max } 3087,3013$, 2924, 2854, 2096, 1589, 1463, 1316, 1262, 1089, 782, $751 \mathrm{~cm}^{-1} ;{ }^{1} \mathrm{H}$ NMR $\left(\mathrm{CDCl}_{3}, 400 \mathrm{MHz}\right): \delta 0.88(\mathrm{t}, 3 \mathrm{H}, J=$ $6.8 \mathrm{~Hz}), 1.25$ (brs, 24H), $1.52-1.56(\mathrm{~m}, 2 \mathrm{H}), 2.63(\mathrm{t}, 1 \mathrm{H}$, $J=7.6 \mathrm{~Hz}), 3.85$ (s, 3H), $4.42(\mathrm{~s}, 2 \mathrm{H}) 6.78(\mathrm{~d}, 1 \mathrm{H}, J=8$ $\mathrm{Hz}), 6.83(\mathrm{~d}, 1 \mathrm{H}, J=8 \mathrm{~Hz}), 7.24-7.26(\mathrm{~m}, 1 \mathrm{H}) ;{ }^{13} \mathrm{C}$ NMR (100 MHz, $\left.\mathrm{CDCl}_{3}\right): \delta 14.09,22.67,29.35,29.48$, 29.56, 29.66, 31.56, 31.91, 32.98, 45.14, 55.49, 108.11, $121.55,121.97,129.27,143.70,158.28 ; \operatorname{ESIMS}(\mathrm{m} / \mathrm{z})$ : $331(\mathrm{M}-\mathrm{N} 3)(\mathrm{M}+\mathrm{H})^{+}$.

Synthesis of 2-methoxy-6-pentadecyl-benzyl amine (7): A solution of compound $6(1.5 \mathrm{~g}, 4.021 \mathrm{mmol})$ in ethanol $(30 \mathrm{ml})$ was taken into a $500 \mathrm{ml}$ Parr-hydrogenation vessel and added a suspension of $10 \% \mathrm{Pd} / \mathrm{C}$ $(250 \mathrm{mg}, 10 \%)$ in $20 \mathrm{ml}$ of ethanol under argon atmosphere and applied $\mathrm{H}_{2}$-pressure (60 psi) for $2 \mathrm{hrs}$. Reaction mixture was filtered through celite bed and concentrated the filtrate under reduced pressure to obtain 2-methoxy-6-pentadecyl-benzyl amine (7) $(1.1 \mathrm{~g}, 78.8 \%)$ as a off-white solid. M.p.: $55-56^{\circ} \mathrm{C}$, IR (neat): $v_{\max } 3400$, 3066, 2924, 2853, 1583, 1464, 1439, 1374, 1259, 1149 , $1121,1079,879,787,742 \mathrm{~cm}^{-1} ;{ }^{1} \mathrm{H}$ NMR $\left(\mathrm{CDCl}_{3}, 400\right.$ $\mathrm{MHz}): \delta 0.89$ (t, 3H, $J=7.2 \mathrm{~Hz}), 1.26$ (brs, 24H), 1.51 $1.57(\mathrm{~m}, 2 \mathrm{H}), 1.76(\mathrm{bs}, 2 \mathrm{H}), 2.65(\mathrm{t}, 2 \mathrm{H}, J=7.6 \mathrm{~Hz}), 3.84$ $(\mathrm{s}, 5 \mathrm{H}), 6.74(\mathrm{~d}, 1 \mathrm{H}, J=8 \mathrm{~Hz}), 6.79(\mathrm{~d}, 1 \mathrm{H}, J=7.6 \mathrm{~Hz})$, $7.15(\mathrm{t}, 1 \mathrm{H}, J=7.6 \mathrm{~Hz}) ;{ }^{13} \mathrm{C}$ NMR $\left(100 \mathrm{MHz}, \mathrm{CDCl}_{3}\right): \delta$ $14.06,22.64,29.31,29.49,29.57,29.61,31.88,32.14$, $33.14,37.54,55.24,107.96,122.02,127.33,129.76$, 141.62, 157.87; MS (m/z): $348(\mathrm{M}+\mathrm{H})^{+}$.

Synthesis of urea and thiourea compounds: A solution of amine (1.0 eq) in dry $\mathrm{CHCl}_{3}$ was taken in seal tube was added isocynate or iso thiocynate and stirred at room temperature for $3 \mathrm{hrs}$ to $8 \mathrm{hrs}$ reaction went to completed distilled off solvent crude compound was purified by column chromatography.

Synthesis of 1-ethyl-3-(2-methoxy-6-pentadecylbenzyl) urea (8a): Using 7 and ethyl isocyanate as starting materials, the title compound $8 \mathrm{a}$ was obtained as a white solid (Yield $=63.1 \%$ ); m.p. $114^{\circ} \mathrm{C}-115^{\circ} \mathrm{C}$; IR (KBr): $v_{\max }$ 3335, 3050, 2967, 2921, 2849, 1622, 1576, 1467, 1260, 1095, 785, $732 \mathrm{~cm}^{-1}$; ${ }^{1} \mathrm{H}$ NMR $\left(\mathrm{CDCl}_{3}, 400 \mathrm{MHz}\right): \delta$ 
$0.88(\mathrm{t}, 3 \mathrm{H}, J=6.8 \mathrm{~Hz}), 1.12(\mathrm{t}, 3 \mathrm{H}, J=7.2 \mathrm{~Hz}), 1.25$ (brs, 24H), $1.48-1.58(\mathrm{~m}, 2 \mathrm{H}), 2.73(\mathrm{t}, 2 \mathrm{H}, J=7.8 \mathrm{~Hz})$, 3.17-3.20 (m, 2H), 3.84 (s, 3H), 4.3 (brs, 1H), 4.40 (d, $2 \mathrm{H}, J=6 \mathrm{~Hz}), 4.65(\mathrm{bs}, 1 \mathrm{H}), 6.73(\mathrm{~d}, 1 \mathrm{H}, J=8.4 \mathrm{~Hz})$, $6.82(\mathrm{~d}, 1 \mathrm{H}, J=8 \mathrm{~Hz}) 7.18(\mathrm{t}, 1 \mathrm{H}, J=7.6 \mathrm{~Hz}$; ESIMS $(\mathrm{m} / \mathrm{z}): 419(\mathrm{M}+\mathrm{H})^{+}$.

Synthesis of 1-cyclopentyl-3-(2-methoxy-6-pentdecylbenzyl) urea (8b): Using 7 and cyclopentyl isocyanate as starting materials, the title compound $8 \mathrm{~b}$ was obtained as a white solid (Yield $=37.8 \%$ ); M.p.: $128^{\circ} \mathrm{C}-129^{\circ} \mathrm{C}$; IR (DCM film): $v_{\max } 3328,2921,2851,1621,1573,1466$, 1260, 1092, $782 \mathrm{~cm}^{-1} ;{ }^{1} \mathrm{H} \mathrm{NMR}\left(\mathrm{CDCl}_{3}, 400 \mathrm{MHz}\right): \delta$ $0.88(\mathrm{t}, 3 \mathrm{H}, J=6.8 \mathrm{~Hz}), 1.24-1.37(\mathrm{~m}, 26 \mathrm{H}), 1.50-1.66$ $(\mathrm{m}, 6 \mathrm{H}), 1.88-1.96(\mathrm{~m}, 2 \mathrm{H}), 2.73(\mathrm{t}, 2 \mathrm{H}, J=7.6 \mathrm{~Hz})$, $3.84(\mathrm{~s}, 3 \mathrm{H}), 3.86-3.95(\mathrm{~m}, 1 \mathrm{H}), 4.32$ (bs, 1H), 4.40 (d, $2 \mathrm{H}, J=5.2 \mathrm{~Hz}), 4.65(\mathrm{~s}, 1 \mathrm{H}), 6.73(\mathrm{~d}, 1 \mathrm{H}, J=8.4 \mathrm{~Hz})$, $6.82(\mathrm{~d}, 1 \mathrm{H}, J=7.6 \mathrm{~Hz}), 7.18(\mathrm{t}, 1 \mathrm{H}, J=7.6 \mathrm{~Hz}) ;{ }^{13} \mathrm{C}$ NMR $\left(100 \mathrm{MHz}, \mathrm{CDCl}_{3}\right): \delta 14.09,22.66,23.56,29.33$, 29.63, 29.66, 31.89, 33.11, 33.58, 35.87, 52.15, 55.43, 107.89, 122.30, 125.19, 128.11, 143.25, 157.71, 157.97; $\operatorname{ESIMS~(m/z):~} 459(\mathrm{M}+\mathrm{H})^{+}$.

Synthesis of 1-(2-methoxy-6-pentadecylbenzyl)-3-phenylurea (8c): Using 7 and phenyl isocyanate as start- ing materials, the title compound $8 \mathrm{c}$ was obtained as a white solid $\left(\right.$ Yield $=58.3 \%$ ); M.p. $105^{\circ} \mathrm{C}-106^{\circ} \mathrm{C}$; IR $(\mathrm{KBr})$ : $v_{\max } 3324,3041,2923,2851,1639,1559,1465,1306$, 1238, 1088, 912, 782, $729 \mathrm{~cm}^{-1} ;{ }^{1} \mathrm{H}$ NMR $\left(\mathrm{CDCl}_{3}, 400\right.$ $\mathrm{MHz}): \delta 0.88(\mathrm{t}, 3 \mathrm{H}, J=6.8 \mathrm{~Hz}), 1.24$ (brs, 24H), 1.54 $1.56(\mathrm{~m}, 2 \mathrm{H}), 2.76(\mathrm{t}, 2 \mathrm{H}, J=7.6 \mathrm{~Hz}), 3.82(\mathrm{~s}, 3 \mathrm{H}), 4.49$ $(\mathrm{d}, 2 \mathrm{H}, \mathrm{J}=5.6 \mathrm{~Hz}), 5.2$ (brs, 1H), 6.25 (bs, 1H), 6.73 (d, $1 \mathrm{H}, J=8.4 \mathrm{~Hz}), 6.82(\mathrm{~d}, 1 \mathrm{H}, J=7.6 \mathrm{~Hz}), 7.05(\mathrm{~m}, 1 \mathrm{H})$ $7.18(\mathrm{t}, 1 \mathrm{H}, J=7.6 \mathrm{~Hz}), 7.24-7.28(\mathrm{~m}, 4 \mathrm{H}) ;{ }^{13} \mathrm{C} \mathrm{NMR}$ (100 MHz, DMSO-d6): $\delta$ 13.90, 22.05, 28.67, 28.87, 29.00, 31.26, 31.56, 32.38, 34.15, 55.54, 108.34, 117.30, $120.80,121.67,124.97,128.11,128.54,140.50,142.29$, 154.71, 157.97; ESIMS (m/z): $467(\mathrm{M}+\mathrm{H})^{+}$.

Synthesis of 1-(3-chlorophenyl)-3-(2-methoxy-6-pentadecylbenzyl)urea (8d): Using 7 and 3-chlorophenyl isocyanate as starting materials, the title compound $8 \mathrm{~d}$ was obtained as a off white solid (Yield $=80.8 \%$ ); M.p. $101^{\circ} \mathrm{C}-102^{\circ} \mathrm{C}$; IR (DCM film): $v_{\max } 3318,2999$, 2922, 2850, 1632, 1582, 1557, 1470, 1260, 1235, 1089, $770 \mathrm{~cm}^{-1} ;{ }^{1} \mathrm{H} \mathrm{NMR}\left(\mathrm{CDCl}_{3}, 400 \mathrm{MHz}\right): \delta 0.87(\mathrm{t}, 3 \mathrm{H}, J=$ $7.2 \mathrm{~Hz}), 1.24$ - 1.36 (brs, 24H), $1.48-1.6(\mathrm{~m}, 2 \mathrm{H}), 2.75(\mathrm{t}$, $2 \mathrm{H}, J=8 \mathrm{~Hz}), 3.86(\mathrm{~s}, 3 \mathrm{H}), 4.50(\mathrm{~d}, 2 \mathrm{H}, J=6 \mathrm{~Hz}), 5.16$ (brs, 1H), 6.3 (brs, 1H), 6.75 (d, 1H, $J=7.6 \mathrm{~Hz}), 6.83$ (d, $1 \mathrm{H}, J=8 \mathrm{~Hz}), 6.99$ (d, 1H, $J=8 \mathrm{~Hz}), 7.125-7.22(\mathrm{~m}$, $3 \mathrm{H}), 7.38(\mathrm{~s}, 1 \mathrm{H})$; ESIMS (m/z): $501(\mathrm{M}+\mathrm{H})^{+}$.

Synthesis of 1-(3,4-dichlorophenyl)-3-(2-methoxy-6pentadecylbenzyl)urea (8e): Using 7 and 3,4-dichlorophenyl isocyanate as starting materials, the title compound $8 \mathrm{e}$ was obtained as a white solid (Yield $=68.1 \%$ ); M.p. $118^{\circ} \mathrm{C}-119^{\circ} \mathrm{C}$; IR (DCM film): $v_{\max } 3355,3288$, 2955, 2920, 2848, 1634, 1582, 1549, 1466, 1381, 1264,
1120, 1088, $776 \mathrm{~cm}^{-1} ;{ }^{1} \mathrm{H}$ NMR $\left(\mathrm{CDCl}_{3}, 400 \mathrm{MHz}\right): \delta$ $1.02(\mathrm{t}, 3 \mathrm{H}, J=7.2 \mathrm{~Hz}), 1.38-1.5(\mathrm{~m}, 24 \mathrm{H}), 1.62-1.67(\mathrm{~m}$, $2 \mathrm{H}), 2.88(\mathrm{t}, 2 \mathrm{H}, J=7.6 \mathrm{~Hz}), 4.01(\mathrm{~s}, 3 \mathrm{H}), 4.63(\mathrm{~d}, 2 \mathrm{H}, J$ $=5.6 \mathrm{~Hz}), 5.25(\mathrm{~s}, 1 \mathrm{H}), 6.5(\mathrm{~s}, 1 \mathrm{H}), 6.91(\mathrm{~d}, 1 \mathrm{H}, J=8 \mathrm{~Hz})$, $6.98(\mathrm{~d}, 1 \mathrm{H}, J=8 \mathrm{~Hz}), 7.29-7.45(\mathrm{~m}, 3 \mathrm{H}), 7.67$ (s, 1H); ${ }^{13} \mathrm{C}$ NMR (100 MHz, DMSO-d6): $\delta$ 13.88, 22.07, 28.69, 28.92, 29.03, 31.26, 31.62, 32.41, 34.16, 55.54, 108.32, $117.30,118.32,121.69,122.02,124.62,128.20,130.30$, 130.93, 140.68, 142.32, 154.28, 157.96; ESIMS (m/z): $535(\mathrm{M}+\mathrm{H})^{+} .537$ (chloro).

Synthesis of 1-(2-fluorophenyl)-3-(2-methoxy-6- pentadecylbenzyl)urea (8f): Using 7 and 2-fluorohenyl isocyanate as starting materials, the title compound $8 \mathrm{f}$ was obtained as a white solid (Yield $=40.5 \%$ ); M.p.: $110^{\circ} \mathrm{C}-111^{\circ} \mathrm{C}$; IR (DCM film): $v_{\max } 3321,3066,3002$, 2923, 2851, 1639, 1601, 1560, 1462, 1310, 1258, 1092, $750 \mathrm{~cm}^{-1} ;{ }^{1} \mathrm{H} \mathrm{NMR}\left(\mathrm{CDCl}_{3}, 400 \mathrm{MHz}\right): \delta 0.87(\mathrm{t}, 3 \mathrm{H}, J=$ $6.8 \mathrm{~Hz}), 1.24-1.36(\mathrm{~m}, 24 \mathrm{H}), 1.51-1.56(\mathrm{~m}, 2 \mathrm{H}), 2.76(\mathrm{t}$, $2 \mathrm{H}, J=8 \mathrm{~Hz}), 3.86(\mathrm{~s}, 3 \mathrm{H}), 4.52(\mathrm{~d}, 2 \mathrm{H}, J=5.6 \mathrm{~Hz}), 5.16$ $(\mathrm{s}, 1 \mathrm{H}), 6.4(\mathrm{~s}, 1 \mathrm{H}), 6.75(\mathrm{~d}, 1 \mathrm{H}, J=8.4 \mathrm{~Hz}), 6.83(\mathrm{~d}, 1 \mathrm{H}$, $J=7.2 \mathrm{~Hz}), 6.85-7.08(\mathrm{~m}, 3 \mathrm{H}), 7.20(\mathrm{t}, 1 \mathrm{H}, J=8 \mathrm{~Hz})$, 8.01-8.10 (m, 1H); ESIMS (m/z): $485(\mathrm{M}+\mathrm{H})^{+}$.

Synthesis of 1-(2-cyanophenyl)-3-(2-methoxy-6-pentadecylbenzyl)urea (8g): Using 7 and 2-cyanophenyl isocyanate as starting materials, the title compound $8 \mathrm{~g}$ was obtained as a white solid (Yield $=45.8 \%$ ); M.p.: $140^{\circ} \mathrm{C}-141^{\circ} \mathrm{C}$; IR (DCM film): $v_{\max } 3325,3037,2918$, 2847, 2225, 1702, 1641, 1550, 1468, 1298, 1240, 1087, $756 \mathrm{~cm}^{-1} ;{ }^{1} \mathrm{H}$ NMR $\left(\mathrm{CDCl}_{3}, 400 \mathrm{MHz}\right): \delta 0.87(\mathrm{t}, 3 \mathrm{H}, J=$ $6.8 \mathrm{~Hz}), 1.24-1.4(\mathrm{~m}, 24 \mathrm{H}), 1.49-1.57(\mathrm{~m}, 2 \mathrm{H}), 2.78(\mathrm{t}$, $2 \mathrm{H}, J=8 \mathrm{~Hz}), 3.89(\mathrm{~s}, 3 \mathrm{H}), 4.53(\mathrm{~d}, 2 \mathrm{H}, J=5.2 \mathrm{~Hz}), 5.39$ $(\mathrm{s}, 1 \mathrm{H}), 6.76-6.83(\mathrm{~m}, 3 \mathrm{H}), 7.02(\mathrm{t}, 1 \mathrm{H}), 7.19-7.21(\mathrm{~m}$, $1 \mathrm{H}), 7.47-7.51(\mathrm{~m}, 2 \mathrm{H}), 8.31(\mathrm{~d}, 1 \mathrm{H}, J=8.4 \mathrm{~Hz})$; ESIMS $(\mathrm{m} / \mathrm{z}): 492(\mathrm{M}+\mathrm{H})^{+}$.

Synthesis of 1-(3-cyanophenyl)-3-(2-methoxy-6-pentadecylbenzyl)urea (8h): Using 7 and 3-cyanophenyl isocyanate as starting materials, the title compound $8 \mathrm{hrs}$ was obtained as a cream color solid (Yield $=70.6 \%$ ); M.p.: $100^{\circ} \mathrm{C}-101^{\circ} \mathrm{C}$; IR (DCM film): $v_{\max } 3324,3196$, 3078, 2921, 2850, 2230, 1636, 1559, 1467, 1239, 1091, $882,786,731 \mathrm{~cm}^{-1} ;{ }^{1} \mathrm{H}$ NMR $\left(\mathrm{CDCl}_{3}, 400 \mathrm{MHz}\right): \delta 0.88$ (t, 3H, $J=6.8 \mathrm{~Hz}), 1.24$ (brs, 24H), $1.45-1.56(\mathrm{~m}, 2 \mathrm{H})$, $2.75(\mathrm{t}, 2 \mathrm{H}, J=8 \mathrm{~Hz}), 3.89(\mathrm{~s}, 3 \mathrm{H}), 4.50(\mathrm{~d}, 2 \mathrm{H}, J=5.2$ $\mathrm{Hz}), 5.14$ (brs, 1H), 6.5 (bs, 1H), 6.77 (d, 1H, $J=8 \mathrm{~Hz}$ ), $6.84(\mathrm{~d}, 1 \mathrm{H}, J=8 \mathrm{~Hz}), 7.19-7.28(\mathrm{~m}, 2 \mathrm{H}), 7.35$ (t, 1H, $J$ $=7.6 \mathrm{~Hz}), 7.55(\mathrm{~d}, 1 \mathrm{H}, J=7.2 \mathrm{~Hz}), 7.69(\mathrm{~s}, 1 \mathrm{H}) ;{ }^{13} \mathrm{C}$ NMR (100 MHz, $\left.\mathrm{CDCl}_{3}\right): \delta 14.08,22.65,29.32,29.54$, 29.59, 29.66, 31.88, 33.10, 35.69, 55.50, 108.03, 112.49, $118.80,121.89,122.39,123.30,124.34,125.81,128.43$, 129.72, 140.26, 143.02, 154.86, 157.93; ESIMS (m/z): $492(\mathrm{M}+\mathrm{H})^{+}$.

Synthesis of 1-(2-methoxy-6-pentadecylbenzyl)-3-(3(tri-fluoromethyl) phenyl) urea (8i): Using 7 and 3-(trifluoromethyl)phenyl isocyanate as starting materials, the 
title compound $8 \mathrm{i}$ was obtained as a off white solid (Yield $=90.9 \%$ ); M.p.: $88^{\circ} \mathrm{C}-89^{\circ} \mathrm{C}$; IR (DCM film): $v_{\max }$ 3323, 3088, 2922, 2851, 1638, 1560, 1454, 1332, 1253, 1169, 1122, 1076, 888, $755 \mathrm{~cm}^{-1} ;{ }^{1} \mathrm{H}$ NMR $\left(\mathrm{CDCl}_{3}, 400\right.$ $\mathrm{MHz}): \delta 0.87(\mathrm{t}, 3 \mathrm{H}, J=6.8 \mathrm{~Hz}), 1.23$ (brs, 24H), 1.48 $1.57(\mathrm{~m}, 2 \mathrm{H}), 2.74(\mathrm{t}, 2 \mathrm{H}, J=7.2 \mathrm{~Hz}), 3.85(\mathrm{~s}, 3 \mathrm{H}), 4.50$ (d, 2H, $J=5.6 \mathrm{~Hz}$ ), 5.19 (brs, 1H), 6.6 (bs, 1H), 6.75 (d, $1 \mathrm{H}, J=8.4 \mathrm{~Hz}), 6.83(\mathrm{~d}, 1 \mathrm{H}, J=7.2 \mathrm{~Hz}), 7.19(\mathrm{t}, 1 \mathrm{H}, J$ $=8 \mathrm{~Hz}), 7.24-7.26(\mathrm{~m}, 1 \mathrm{H}), 7.36(\mathrm{t}, 1 \mathrm{H}, J=8 \mathrm{~Hz}), 7.52-$ $7.56(\mathrm{~m}, 2 \mathrm{H}) ; \operatorname{ESIMS}(\mathrm{m} / \mathrm{z}): 535(\mathrm{M}+\mathrm{H})^{+}$.

Synthesis of 1-(2-methoxy-6-pentadecylbenzyl)-3-(3methoxyphenyl) urea (8j): Using 7 and 3-methoxyphenyl isocyanate as starting materials, the title compound $8 \mathrm{j}$ was obtained as a off white solid (Yield = 69.9\%); M.p.: $77^{\circ} \mathrm{C}-78^{\circ} \mathrm{C}$; IR (DCM film): $v_{\max } 3318$, 2997, 2923, 2851, 1629, 1604, 1562, 1467, 1264, 1212, $1165,1091,844,779 \mathrm{~cm}^{-1} ;{ }^{1} \mathrm{H}$ NMR $\left(\mathrm{CDCl}_{3}, 400 \mathrm{MHz}\right): \delta$ 0.88 (t, 3H, $J=6.8 \mathrm{~Hz}), 1.24$ (brs, 24H), $1.51-1.57$ (m, $2 \mathrm{H}), 2.75(\mathrm{t}, 2 \mathrm{H}, J=8 \mathrm{~Hz}), 3.76(\mathrm{~s}, 3 \mathrm{H}), 3.82(\mathrm{~s}, 3 \mathrm{H})$, $4.49(\mathrm{~d}, 2 \mathrm{H}, J=5.2 \mathrm{~Hz}), 5.27(\mathrm{t}, 1 \mathrm{H}, J=5.6 \mathrm{~Hz}), 6.31(\mathrm{~s}$, $1 \mathrm{H}), 6.59-6.62(\mathrm{~m}, 1 \mathrm{H}), 6.72-6.82(\mathrm{~m}, 3 \mathrm{H}), 6.94(\mathrm{~s}, 1 \mathrm{H})$, 7.14 - $7.20(\mathrm{~m}, 2 \mathrm{H})$; ESIMS(m/z): $497(\mathrm{M}+\mathrm{H})^{+}$.

Synthesis of 1-(2,2-dimethyl-2,3-dihydrobenzofuran-7yl)-3-(2-methoxy-6- pentadecylbenzyl) urea (8k): Using 7 and 2,2-dimethyl-2,3-dihydrobenzofuran-7yl isocyanate as starting materials, the title compound $8 \mathrm{k}$ was obtained as a light yellow solid (Yield $=66.8 \%$ ); M.p.: $112^{\circ} \mathrm{C}-113^{\circ} \mathrm{C}$; IR (DCM film): $v_{\max } 3356,3299,3045$, 2923, 2854, 1692, 1635, 1556, 1448, 1323, 1304, 1263, 1228, 1132, 1099, 1056, 879,759 $\mathrm{cm}^{-1} ;{ }^{1} \mathrm{H} \mathrm{NMR}\left(\mathrm{CDCl}_{3}\right.$, $400 \mathrm{MHz}): \delta 0.87(\mathrm{t}, 3 \mathrm{H}, J=7.2 \mathrm{~Hz}), 1.23-1.36(\mathrm{~m}$, 24H), $1.42(\mathrm{~s}, 6 \mathrm{H}), 1.49-1.55(\mathrm{~m}, 2 \mathrm{H}), 2.75(\mathrm{t}, 2 \mathrm{H}, J=8$ $\mathrm{Hz}$ ), 3.00 (s, 2H), 3.83 (s, 3H), 4.50 (d, 2H, $J=5.2 \mathrm{~Hz})$, 5.16 (bs, 1H), 6.13 (brs, 1H), $6.72-6.82(\mathrm{~m}, 4 \mathrm{H}), 7.17(\mathrm{t}$, $1 \mathrm{H}, J=8.4 \mathrm{~Hz}), 7.60$ (d, 1H, $J=7.6 \mathrm{~Hz})$; ESIMS (m/z): $537(\mathrm{M}+\mathrm{H})^{+}$.

Synthesis of 1-(2-methoxy-6-pentadecylbenzyl)-3-phenylthiourea (9a): Using 7 and phenyl thioisocyanate as starting materials, the title compound 9a was obtained as a off white solid (Yield $=86.3 \%$ ); M.p.: $71^{\circ} \mathrm{C}-72^{\circ} \mathrm{C}$; IR (DCM film): $v_{\max } 3387,3192,3005,2924,2853,1590$, 1529, 1461, 1309, 1253, 1171, 1081, 750, $695 \mathrm{~cm}^{-1} ;{ }^{1} \mathrm{H}$ $\operatorname{NMR}\left(\mathrm{CDCl}_{3}, 400 \mathrm{MHz}\right): \delta 0.88(\mathrm{t}, 3 \mathrm{H}, J=6.8 \mathrm{~Hz}), 1.25$ - $1.38(\mathrm{~m}, 24 \mathrm{H}), 1.53-1.57(\mathrm{~m}, 2 \mathrm{H}), 2.83(\mathrm{t}, 2 \mathrm{H}, \mathrm{J}=8$ $\mathrm{Hz}), 3.65$ (s, 3H), 4.92 (d, 2H, J = 3.6 Hz), 6.67 (d, 1H, J $=8 \mathrm{~Hz}), 6.82(\mathrm{~d}, 1 \mathrm{H}, J=7.6 \mathrm{~Hz}), 6.9(\mathrm{bs}, 1 \mathrm{H}), 7.13-7.19$ (m, 3H), $7.23-7.29(\mathrm{~m}, 1 \mathrm{H}), 7.36-7.40(\mathrm{~m}, 2 \mathrm{H}), 7.52(\mathrm{~s}$, $1 \mathrm{H}) ; \operatorname{ESIMS}(\mathrm{m} / \mathrm{z}): 483(\mathrm{M}+\mathrm{H})^{+}$.

Synthesis of 1-(3-chlorophenyl)-3-(2-methoxy-6- pentadecylbenzyl)thiourea (9b): Using 7 and 3-chloro phenyl thioisocyanate as starting materials, the title compound $9 \mathrm{~b}$ was obtained as a off white solid (Yield = 76.6\%); M.p.: $81^{\circ} \mathrm{C}-82^{\circ} \mathrm{C}$; IR (DCM film): $v_{\max } 3332$, 3089, 3029, 2921, 2849, 1634, 1583, 1558, 1469, 1259,
1088, 894, 860, $779 \mathrm{~cm}^{-1} ;{ }^{1} \mathrm{H}$ NMR $\left(\mathrm{CDCl}_{3}, 400 \mathrm{MHz}\right)$ : $\delta 0.88(\mathrm{t}, 3 \mathrm{H}, J=6.8 \mathrm{~Hz}), 1.24-1.40(\mathrm{~m}, 24 \mathrm{H}), 1.50-$ $1.56(\mathrm{~m}, 2 \mathrm{H}), 2.75(\mathrm{t}, 2 \mathrm{H}, J=8 \mathrm{~Hz}), 3.85(\mathrm{~s}, 3 \mathrm{H}), 4.49(\mathrm{~d}$, $2 \mathrm{H}, J=6 \mathrm{~Hz}), 5.2(\mathrm{~s}, 1 \mathrm{H}), 6.40(\mathrm{~s}, 1 \mathrm{H}), 6.75(\mathrm{~d}, 1 \mathrm{H}, J=$ $8 \mathrm{~Hz}), 6.82(\mathrm{~d}, 1 \mathrm{H}, J=7.2 \mathrm{~Hz}), 6.98-7.02(\mathrm{~m}, 1 \mathrm{H}), 7.12$ - 7.22 (m, 3H), 7.36-7.39 (m, 1H); ${ }^{13} \mathrm{C} \mathrm{NMR} \mathrm{(100} \mathrm{MHz,}$ $\left.\mathrm{CDCl}_{3}\right) \delta: 14.11,22.68,29.35,29.62,29.69,31.91,33.15$, $35.81,55.50,108.00,117.91,119.89,122.41,122.99$, $124.52,128.37,129.95,134.59,140.28,143.15,154.98$, 157.96; ESIMS (m/z): $517(\mathrm{M}+\mathrm{H})^{+}$.

Synthesis of 1-(3,4-dichlorophenyl)-3-(2-methoxy-6pentadecylbenzyl) thiourea (9c): Using 7 and 3,4-dichloro phenyl thioisocyanate as starting materials, the title compound 9c was obtained as a off white solid (Yield $=73.5 \%$ ); M.p.:92 ${ }^{\circ} \mathrm{C}-93^{\circ} \mathrm{C}$; IR (DCM film): $v_{\max }$ 3262, 3052, 3003, 2924, 2853, 1587, 1529, 1469, 1313, $1255,1128,1084,1031,775 \mathrm{~cm}^{-1} ;{ }^{1} \mathrm{H}$ NMR $\left(\mathrm{CDCl}_{3}, 400\right.$ $\mathrm{MHz}): \delta 0.88(\mathrm{t}, 3 \mathrm{H}, J=6.8 \mathrm{~Hz}), 1.25-1.40(\mathrm{~m}, 24 \mathrm{H})$, $1.52-1.60(\mathrm{~m}, 2 \mathrm{H}), 2.8$ (brs, 2H), 3.77 (s, 3H), $4.90(\mathrm{~s}$, 2H), $6.72-7.01(\mathrm{~m}, 4 \mathrm{H}), 7.18-7.3(\mathrm{~m}, 2 \mathrm{H}), 7.41-7.51$ (m, 2H); ESIMS (m/z): $551(\mathrm{M}+\mathrm{H})^{+} .553$ (chloro).

Synthesis of 1-(2-fluorophenyl)-3-(2-methoxy-6-pentadecylbenzyl)thiourea (9d): Using 7 and 2-fluoro-phenyl thioisocyanate as starting materials, the title compound 9d was obtained as a off white solid (Yield = 87.9\%); M.p.: $80^{\circ} \mathrm{C}-81^{\circ} \mathrm{C}$; IR (DCM film): $v_{\max } 3276$, 3224, 3000, 2923, 2852, 1585, 1531, 1462, 1318, 1257, 1085, 860, $752 \mathrm{~cm}^{-1} ;{ }^{1} \mathrm{H} \mathrm{NMR}\left(\mathrm{CDCl}_{3}, 400 \mathrm{MHz}\right): \delta$ $0.88(\mathrm{t}, 3 \mathrm{H}, J=6.8 \mathrm{~Hz}), 1.25-1.40(\mathrm{~m}, 24 \mathrm{H}), 1.51-1.60$ $(\mathrm{m}, 2 \mathrm{H}), 2.8(\mathrm{bs}, 2 \mathrm{H}), 3.69(\mathrm{~s}, 3 \mathrm{H}), 4.91(\mathrm{~s}, 2 \mathrm{H}), 6.63-$ $6.66(\mathrm{~m}, 1 \mathrm{H}), 6.82-6.90(\mathrm{~m}, 2 \mathrm{H}), 7.14-7.3(\mathrm{~m}, 5 \mathrm{H})$; $\operatorname{ESIMS~(m/z):~} 501(\mathrm{M}+\mathrm{H})^{+}$.

Synthesis of 1-(2-methoxy-6-pentadecylbenzyl)-3- cyano phenylthiourea (9e): Using 7 and 3-cyano phenyl thioisocyanate as starting materials, the title compound $9 \mathrm{e}$ was obtained as a yellow solid (Yield $=82.1 \%$ ); M.p.: $81^{\circ} \mathrm{C}-82^{\circ} \mathrm{C}$; IR (DCM film): $v_{\max } 3349,2929,2853$, 2228, 1587, 1529, 1463, 1258, 1085, $757 \mathrm{~cm}^{-1}$; ${ }^{1} \mathrm{H}$ NMR $\left(\mathrm{CDCl}_{3}, 400 \mathrm{MHz}\right): \delta 0.88(\mathrm{t}, 3 \mathrm{H}, J=6.8 \mathrm{~Hz}), 1.25-1.38$ (m, 24H), $2.50-2.60(\mathrm{~m}, 2 \mathrm{H}), 2.79$ (bs, 2H), $3.78(\mathrm{~s}, 3 \mathrm{H})$, $4.92(\mathrm{~s}, 2 \mathrm{H}), 6.74(\mathrm{~d}, 1 \mathrm{H}, J=7.6 \mathrm{~Hz}), 6.85(\mathrm{~d}, 1 \mathrm{H}, J=7.2$ $\mathrm{Hz}), 7.20-7.29(\mathrm{~m}, 3 \mathrm{H}), 7.55$ - 7.66 (m, 3H); ESIMS $(\mathrm{m} / \mathrm{z}): 508(\mathrm{M}+\mathrm{H})^{+}$.

Synthesis of 1-(2-methoxy-6-pentadecylbenzyl)-3-(3(trifluoromethyl) phenyl) thiourea (9f): Using 7 and 3(trifluoromethyl)phenyl thioisocyanate as starting materials, the title compound 9f was obtained as a off white solid (Yield $=84.2 \%$ ); IR (DCM film): $v_{\max } 3374,3204$, 2922, 2852, 1586, 1530, 1459, 1328, 1256, 1126, 1075, 894, $780 \mathrm{~cm}^{-1}$; ${ }^{1} \mathrm{H}$ NMR $\left(\mathrm{CDCl}_{3}, 400 \mathrm{MHz}\right): \delta 0.88(\mathrm{t}$, $3 \mathrm{H}, J=6.8 \mathrm{~Hz}), 1.25-1.38(\mathrm{~m}, 24 \mathrm{H}), 1.54-1.56(\mathrm{~m}$, 2H), 2.82 (brs, 2H), 3.69 (s, 3H), 4.92 (s, 2H), 6.65-6.84 $(\mathrm{m}, 3 \mathrm{H}), 7.18$ - $7.21(\mathrm{~m}, 1 \mathrm{H}), 7.28$ - $7.6(\mathrm{~m}, 4 \mathrm{H})$; ESIMS $(\mathrm{m} / \mathrm{z}): 551(\mathrm{M}+\mathrm{H})^{+}$. 
Antibacterial Bioassay [26]: Urea and thiourea derivatives of Anacardic acid (8a - 8k, 9a - 9f) were dissolved in dimethyl sulphoxide at $250 \mu \mathrm{g} / \mathrm{ml}$ concentration. The composition of nutrient agar medium was Bactotryptone $(10 \mathrm{~g})$, yeast extract $(5 \mathrm{~g}), \mathrm{NaCl}(10 \mathrm{~g})$, final $\mathrm{pH}$ 7.4. After $18 \mathrm{~h}$ the exponentially growing cultures of the six bacteria in nutrient broth at $37^{\circ} \mathrm{C}$ were diluted in sterile broth. From each of these diluted cultures, $1 \mathrm{~mL}$ was added to $100 \mathrm{~mL}$ sterilized and cooled nutrient agar media to give a final bacterial count of $1 \times 10^{6} \mathrm{cell} / \mathrm{ml}$. The plates were set at room temperature and later dried at $37^{\circ} \mathrm{C}$ for $20 \mathrm{hrs}$. Paper discs $(6 \mathrm{~mm}$, punched from Whatmann no. 41 paper) were ultraviolet sterilized and used for the assays. Discs were soaked in different concentration of the test solution and placed on the inoculated agar media at regular intervals of $6-7 \mathrm{~cm}$, care was taken to ensure that excess solution was not on the discs. All the samples were taken in triplicates. The plates were incubated at $37^{\circ} \mathrm{C}$ in an inverted fashion. Activity was determined by zones showing complete inhibition $(\mathrm{mm})$. Growth inhibition was calculated with reference to positive control.

\section{Acknowledgements}

We thank GVK Biosciences Private Limited for the financial support and encouragement. We also thankful to the analytical department for their analytical data and to Dr. Balaram Patro for his helpful suggestions.

\section{REFERENCES}

[1] J. H. P. Tyman, "Non-Isoprenoid Long Chain Phenols," Chemical Society Reviews, Vol. 8, No. 4, 1979, pp. 499537. doi:10.1039/cs9790800499

[2] I. Kubo, M. Ochi, P. C. Viera and S. Komatsu, "Antitumor Agents from the Cashew (Anacardium occidentale) apple juice," Journal of Agricultural and Food Chemistry, Vol. 41, No. 6, 1993, pp. 1012-1015. doi:10.1021/jf00030a035

[3] M. J. Rybak and R. L. Akins, "Emergence of Methicillin-Resistant Staphylococcus Aureus with Intermediate Glycopeptide Resistance: Clinical Significance and Treatment Options," Drugs, Vol. 61, No. 1, 2001, pp. 1-7. doi:10.2165/00003495-200161010-00001

[4] M. Lipsitch, "The Rise and Fall of Antimicrobial Resistance," Trends Microbiology, Vol. 9, No. 9, 2001, pp. 438-444. doi:10.1016/S0966-842X(01)02130-8

[5] B. A. Cunha, Drugs Today, Vol. 34, 1998, pp. 691-698.

[6] A. Marchese, G. C. Schito and E. A. Debbia, "Evolution of Antibiotic Resistance in Gram-Positive Pathogens," Journal of Chemotherapy, Vol. 12, No. 6, 2000, pp. 459462.

[7] Y. Cetinkaya, P. Falk and C. G. Mayhall, "Vancomycin-Resistant Enterococci," Clinical Microbiology Reviews,
Vol. 13, No. 4, 2000, pp. 686-707. doi:10.1128/CMR.13.4.686-707.2000

[8] G. H. Cassell and J. Mekalanos, "Development of Antimicrobial Agents in the Era of New and Reemerging Infectious Diseases and Increasing Antibiotic Resistance," Journal of the American Medical Association, Vol 285, No. 5, 2001, pp. 601-605. doi:10.1001/jama.285.5.601

[9] D. T. W. Chu, J. J. Plattner and L. Katz, "New Directions in Antibacterial Research," Journal of Medicinal Chemistry, Vol. 39, No. 20, 1996, pp. 3853-3874. doi:10.1021/jm960294s

[10] I. Kubo, H. Muroi and M. Himejima, "Structure-Anti Bacterial Activity Relationships of Anacardic Acids," Journal of Agricultural and Food Chemistry, Vol. 41, No. 6, 1993, pp. 1016-1019. doi:10.1021/jf00030a036

[11] J. L. Gillerman, N. J. Walsh, N. K. Werner and H. Schlenk, "Antimicrobial Effects of Anacardic Acids," Canadian Journal of Microbiology, Vol. 15, No. 10, 1969, pp. 12191223. doi:10.1139/m69-220

[12] A. S. Gulati and B. C. Subba Rao, Indian Journal of Chemistry, Vol. 2, 1964, pp.337.

[13] M. A. EIShol, P. D. Adawadkar, D. A. Benign, E. S. Watson and T. L. Little Jr., "6-(Alkylamino)-9-benzyl9H-purines. A New Class of Anticonvulsant Agents," Journal of Medicinal Chemistry, Vol. 29, 1986, pp. 606612.

[14] S. V. Shoba, C. S. Ramadoss and B. Ravindranath, "Inhibition of Soybene Lipoxygenase-1 by Anacardic Acids, Cardol, and Cardanols," Journal of Natural Products, Vol. 57, No. 12, 1994, pp. 1755-1757. doi: $10.1021 / \mathrm{np} 50114 \mathrm{a} 025$

[15] T. J. Ha and I. Kubo, "Lipoxygenase Inhibitory Activity of Anacardic Acids," Journal of Agricultural and Food Chemistry, Vol. 53, No. 11, 2005, pp.4350-4354. doi:10.1021/jf048184e

[16] V. Chandregowda, A. Kush and G. C. Reddy, "Synthesis of Benzamide Derivatives of Anacardic Acid and Their Cytotoxic Activity," European Journal of Medicinal Chemistry, Vol. 44, No. 6, 2009, pp. 2711-2719. doi:10.1016/j.ejmech.2009.01.033

[17] R. Paramashivappa, P. Phanikumar, P. V. Subba Rao and A. Srinivasa Rao, "Synthesis of Sildenafil Analogues from Anacardic Acid and Their Phosphodiesterase-5 Inhibition," Journal of Agricultural and Food Chemistry, Vol. 50, No. 26, 2002, pp. 7709-7713. doi: $10.1021 / \mathrm{jf0258050}$

[18] P. Phanikumar, S. C. Stotz, R. Paramashivappa, M. Beedle, G. W. Zamponi and A. Srinivasa Rao, "Synthesis and Evaluation of a New Class of Nifedipine Analogs with T-Type Calcium Channel Blocking Activity," Molecular Pharmacology, Vol. 61, No. 3, 2002, pp. 649-658. doi: $10.1124 / \mathrm{mol} .61 .3$

[19] B. Narayana Swamy, T. K. Suma, G. Venkateswara Rao and G. Chandrasekara Reddy, European Journal of Medicinal Chemistry, Vol. 42, 2007, pp. 422-424.

[20] K. Mantelingu, A. H. Kishore, K. Balasubramanyam, G. V. Kumar, M. Altaf, S. N. Swamy, R. Selvi, C. Das, C. 
Narayana, K. S. Rangappa and T. K. Kundu, "Activation of p300 Histone Acetyltransferase by Small Molecules Altering Enzyme Structure: Probed by Surface-Enhanced Raman Spectroscopy," Journal of Physical Chemistry $B$, Vol. 111, No. 17, 2007, pp. 4527-4534. doi:10.1021/jp067655s

[21] A. Hari Kishore, B. M. Vedamurth, K. Mantelingu, S. Agrawal, B. A. Ashok Redd, S. Roy, K. S. Rangappa and T. K. Kundu, "Specific Small-Molecule Activator of Aurora Kinase A Induces Autophosphorylation in a CellFree System," Journal of Medicinal Chemistry, Vol. 51, No. 4, 2008, pp. 792-797. doi:10.1021/jm700954w

[22] B. Sung, M. K. Pande, K. S. Ahn, T. Yi, M. M. Chaturved, M. Liu and B. B. Aggarwal, "Anacardic Acid (6-Nonadecyl Salicylic Acid), an Inhibitor of Histone Acetyltransferase, Suppresses Expression of Nuclear Factor- $\kappa B-R e g u l a t e d$ Gene Products Involved in Cell Survival, Proliferation, Invasion, and Inflammation through Inhibition of the Inhibitory Subunit of Nuclear Factor$\kappa \mathrm{B} \alpha$ Kinase, Leading to Potentiation of Apoptosis," Blood, Vol. 111, No. 10, 2008, pp. 4880-4891. doi:10.1182/blood-2007-10-117994
[23] L. Cui, J. Miao, T. Furuya, Q. Fan, X. Li, P. K. Rathod, X. Z. Su and L. Cui, "Histone Acetyltransferase Inhibitor Anacardic Acid Causes Changes in Global Gene Expression during in Vitro Plasmodium Falciparum Development," Eukaryotic Cell, Vol.7, No. 7, 2008, pp. 12001210. doi:10.1128/EC.00063-08

[24] G. Sbardella, S. Castellano, C. Vicidomini, D. Rotili, A. Nebbioso, M. Miceli, L. Altucci and A. Mai, "Identification of Long Chain Alkylidenemalonates as Novel Small Molecule Modulators of Histone Acetyl-Transferases," Bioorganic \& Medicinal Chemistry Letters, Vol. 18, No. 9, 2008, pp. 2788-2792. doi:10.1016/j.bmcl.2008.04.017

[25] R. Paramashivappa, P. Phanikumar, P. J. Vithayathil and A. Srinivasa Rao, "Novel Method for Isolation of Major Phenolic Constituents from Cashew (Anacardium occidentale L.) Nut Shell Liquid," Journal of Agricultural and Food Chemistry, Vol. 49, No. 5, 2001, pp. 2548-2551. doi:10.1021/jf001222j

[26] A. Rahman, M. L. Choudhary and W. J. Thomson, "Bioassay Techniques for Drug Development," Harwood Academic Publishers, The Netherlands, 2001. doi: $10.4324 / 9780203304532$ 